\title{
In silico fragmentation for computer assisted identification of metabolite mass spectra
}

\author{
Sebastian Wolf ${ }^{*}$, Stephan Schmidt ${ }^{1}$, Matthias Müller-Hannemann ${ }^{2}$, Steffen Neumann ${ }^{1}$
}

\begin{abstract}
Background: Mass spectrometry has become the analytical method of choice in metabolomics research. The identification of unknown compounds is the main bottleneck. In addition to the precursor mass, tandem MS spectra carry informative fragment peaks, but the coverage of spectral libraries of measured reference compounds are far from covering the complete chemical space. Compound libraries such as PubChem or KEGG describe a larger number of compounds, which can be used to compare their in silico fragmentation with spectra of unknown metabolites.

Results: We created the MetFrag suite to obtain a candidate list from compound libraries based on the precursor mass, subsequently ranked by the agreement between measured and in silico fragments. In the evaluation MetFrag was able to rank most of the correct compounds within the top 3 candidates returned by an exact mass query in KEGG. Compared to a previously published study, MetFrag obtained better results than the commercial MassFrontier software. Especially for large compound libraries, the candidates with a good score show a high structural similarity or just different stereochemistry, a subsequent clustering based on chemical distances reduces this redundancy. The in silico fragmentation requires less than a second to process a molecule, and MetFrag performs a search in KEGG or PubChem on average within 30 to 300 seconds, respectively, on an average desktop PC.

Conclusions: We presented a method that is able to identify small molecules from tandem MS measurements, even without spectral reference data or a large set of fragmentation rules. With today's massive general purpose compound libraries we obtain dozens of very similar candidates, which still allows a confident estimate of the correct compound class. Our tool MetFrag improves the identification of unknown substances from tandem MS spectra and delivers better results than comparable commercial software. MetFrag is available through a web application, web services and as java library. The web frontend allows the end-user to analyse single spectra and browse the results, whereas the web service and console application are aimed to perform batch searches and evaluation.
\end{abstract}

\section{Background}

Mass spectrometry has become the analytical method of choice in metabolomics research [1]. Various ionisation methods are commonly used, such as electron impact (EI) used with gas chromatography (GC/MS), or the soft electrospray ionisation (ESI), which is employed in LC/ ESI-MS systems. The main bottleneck in the interpretation of metabolomics experiments is the identification of compounds. In addition to the exact mass, tandem MS spectra provide additional structural hints, providing a

\footnotetext{
* Correspondence: swolf@ipb-halle.de

'Leibniz Institute of Plant Biochemistry- Department of Stress- and Developmental Biology, Weinberg 3, 06120 Halle(Saale), Germany
}

fingerprint of the measured molecule. In tandem MS, the molecules are interacting with a collision gas at specified kinetic energies, hence the name collision induced dissociation. Large spectral libraries of measured reference spectra exist for GC/MS, such as the commercial NIST library '08 (Gaithersburg, MD) or the GMD [2], but for ESI-tandem MS spectral libraries are still few and comparably small $[3,4]$. A different approach towards identification is the interpretation of the measured spectra, usually with regard to the known (or hypothetical) molecular structure.

Fragmenter with a rule set like the commercial tools ACD Fragmenter [5] and Mass Frontier [6] generate
C Biomed Central

(C) 2010 Wolf et al; licensee BioMed Central Ltd. This is an Open Access article distributed under the terms of the Creative Commons Attribution License (http://creativecommons.org/licenses/by/2.0), which permits unrestricted use, distribution, and reproduction in any medium, provided the original work is properly cited. 
fragments based on cleavage rules known from the literature, in both cases the algorithmic details are not published. For some compounds, MassFrontier 5 is not able to identify any fragments in negative mode [7]. Hill et al. used Mass Frontier 4 to predict the tandem MS spectra of 102 test compounds, which were analysed using a Micromass Q-TOF II in positive mode, to identify the measured compound and its structure. Candidate compounds were retrieved from PubChem using the exact mass. MassFrontier used those structures as input and generated spectra which were compared to the measured spectra. Finally, the compounds were ranked according to the peaks common to both the predicted and measured spectra [8]. Combinatorial Fragmenter such as Fragment Identificator (FiD) proposed by Heinonen et al. [9] try to predict the fragmentation tree given both a metabolite's molecular structure and its tandem mass spectrum. Due to high computational complexity, even for a single medium sized compound (around $300 \mathrm{Da}$ ) runtimes can reach several hours. Another approach is the systematic bond disconnection method without a rule set as described in [10]. The resulting product ions from a single precursor structure are matched against the peaks measured with a high-resolution mass spectrometer. The software EPIC was tested against two hand annotated spectra from the literature and is not publicly available. The runtime was reported to be around 1 minute to process 1-(3-(5-(1,2,4-triazol-4-yl)$1 \mathrm{H}$-indol-3-yl)propyl)-4-(2-(3-fluorophenyl)ethyl)piperazine (432 Da).
MetFrag is a combinatorial fragmenter using the bond disconnection approach, which is fast enough to screen dozens to thousands of candidates retrieved from e.g. KEGG, PubChem or ChemSpider compound databases. We do not attempt to create a mechanistically correct prediction of the fragmentation processes. Instead, we want to perform a search in compound libraries using the measured fragments as additional structural hints.

The paper is structured as follows: in the next section we describe the architecture and the in silico fragmentation algorithm, including heuristics to speed up calculations and to account for molecular rearrangements upon fragmentation. Afterwards, we explain the scoring function. In the results section we evaluate MetFrag on a set of 710 spectra from 151 compounds. The paper finishes with our conclusions. All detailed results are available as additional files.

\section{Implementation}

The workflow implemented in MetFrag is shown in Figure 1, and covered in detail in the following sections. MetFrag is implemented in Java and uses the Chemistry Development Kit [11], an open source Java library. The CDK provides algorithms and data structures for structural Chemo- and Bioinformatics and is able to read and write common formats such as MDL, CML, InChI, and many more.

\section{Retrieval of candidates from compound libraries}

First we perform a search in a general purpose compound database for candidate molecules based on the

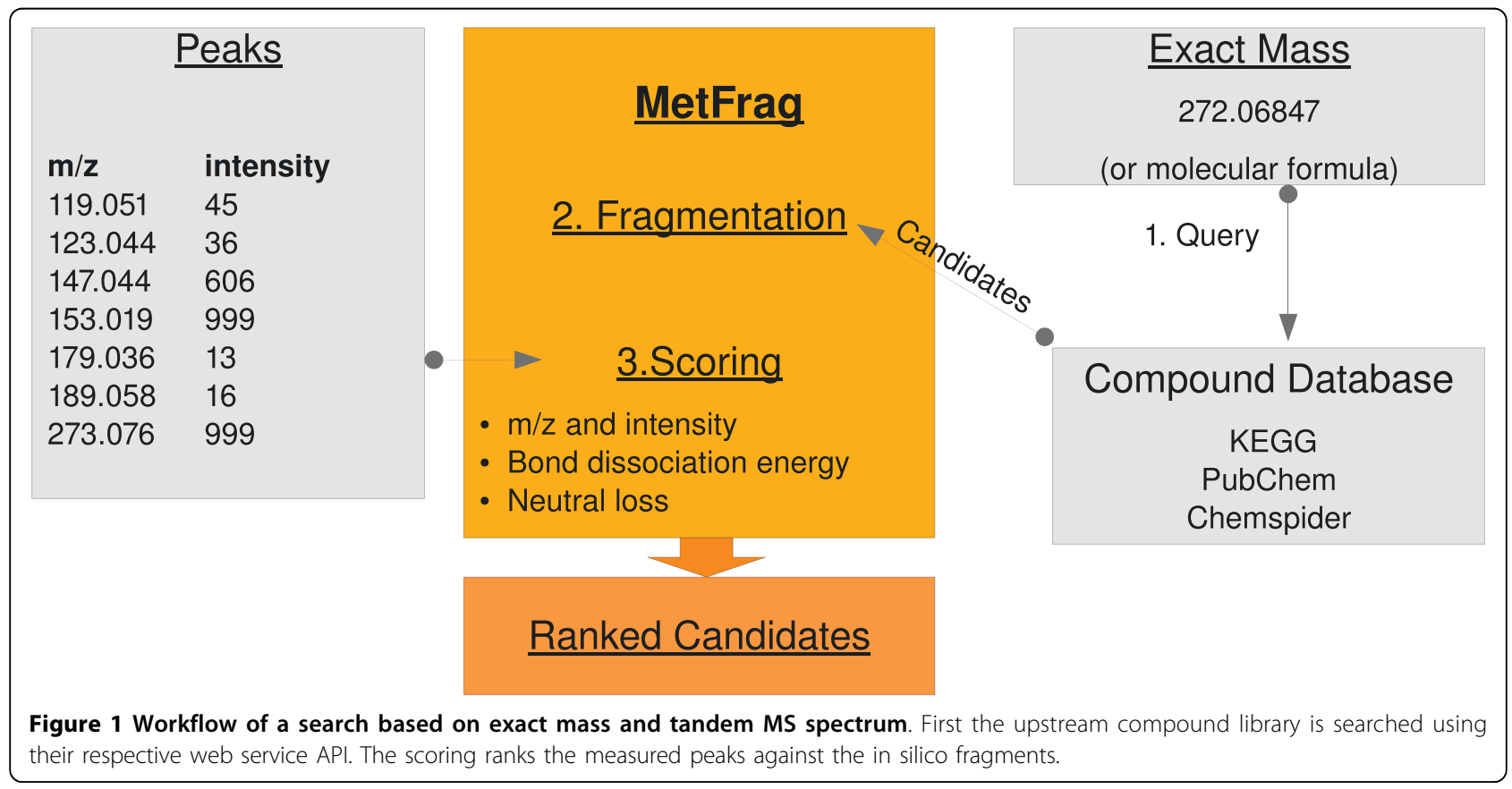


exact mass (within an error range given in ppm) of the neutral and intact molecule. Currently three compound databases can be queried: KEGG Compound (about 16021 entries, October 2009) [12], PubChem (37 million, June 2009) [13] and ChemSpider (23 million, October 2009) [14]. Optionally, the search can be restricted to compounds containing only the elements CHNOPS, commonly occurring in natural products.

Alternatively, the compound databases can be searched with the elemental composition if this has been derived from e.g. exact mass and isotopic pattern of the precursor. Finally, the set of candidates can be supplied by simply enumerating all database IDs to be processed, e.g. obtained by an independent search for metabolites of a pathway. To query other (local) libraries, a custom wrapper can be added which contains the search logic.

The results usually contain dozens to thousands of hits with a similar (or identical in case of isomeric compounds) mass. The databases are accessed via their webservice interface and the resulting candidate compounds are downloaded automatically. Hydrogens are added explicitly to the structure where necessary.

\section{In silico fragmentation of candidates}

MetFrag generates all possible topological fragments of a candidate compound in order to match the fragment mass with the measured peaks. The problem of enumerating all possible molecular fragments can be solved by creating a fragmentation tree. The root consists of the intact molecule, and each node represents a fragment, obtained by splitting the molecule at a given bond. We implemented this as an iterative, breadth-first algorithm. One major speed determining factor is the number of fragments generated, because of the combinatorial nature of the algorithm. Thus, the maximum tree depth was introduced to improve the performance and specificity. We perform additional application-specific steps to prune the search space and take care of molecular rearrangements, see below. For each candidate structure the fragments are generated in the following way (Figure 2):

Initially the candidate structure is pushed into an "unprocessed" queue. The candidate structure is preprocessed using a (small) set of rules, which describe molecular rearrangements during the CID fragmentation that can not be accounted for by the simple bond disconnection approach. Each application of these rules results in one or more derived fragments which are added to the

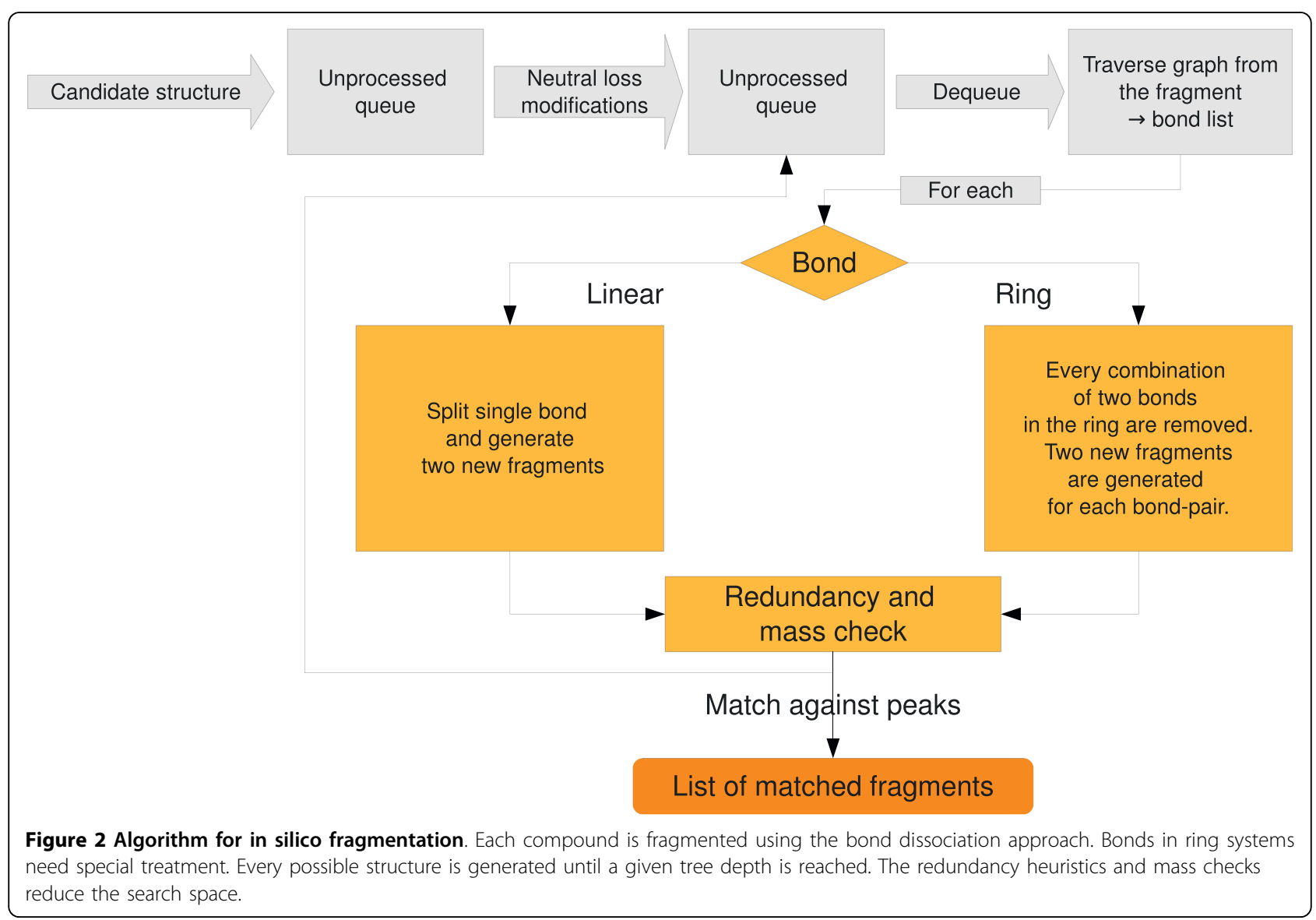


"unprocessed" queue. The actual rules will be described later in this paper.

Then a structure is dequeued and its molecular graph is traversed to collect all bonds to be split. A linear bond (which is not part of a ring system) only needs to be cleaved and results in two new fragments. Within a ring system two bonds have to be split simultaneously, to create the new fragments. Only the fragments larger than the peak with the smallest mass are created, since smaller fragments can not explain an experimental peak.

Before proceeding to the next fragment, a redundancy check is performed to eliminate duplicate fragments. Redundancy occurs if a fragment $A$ is part of both parent fragments $A B$ and $A B C$, or the fragment $A$ appears in different places of the molecule, as in $A B A$. In both cases the redundant structures would cause longer runtimes and higher memory consumption without gaining any information. In addition to full (and time consuming) graph isomorphism checks we describe simpler heuristics later in this paper.

Finally, the in silico fragments are matched against the query peaklist. The measured peaks correspond to the charged fragments, so the matching function adds (positive mode) or removes (negative mode) a proton (1.007 $\mathrm{Da})$ to the fragment mass. In a few cases, fragment ions can have an intrinsic charge, where one of the heteroatoms is charged. In this case the fragment mass is used as-is, but a penalty is added to the bond dissociation energy of this fragment (see below).

The accuracy of a mass measured by an MS instrument is typically expressed relatively in $\mathrm{ppm}$. In practice we found that especially for low masses, an additional (absolute) deviation has to be considered. Hence MetFrag uses two values mzppm and mzabs respectively, to calculate the mass error used for fragment matching.

Peaks that have such an explanation are subsequently removed from the query peaklist and the fragment-peak pair is saved for the final scoring. If the peak with the smallest mass has been explained, this will raise the minimal-mass cut-off, resulting in even fewer fragments that need to be considered. The "unprocessed" queue is then populated with the created and filtered fragments and processed as described above. The fragmentation terminates if the queue is empty or the maximum tree depth has been reached. The candidate is then scored based on all matched fragment-peak pairs as explained in the following section.

\section{Scoring candidates based on fragments explaining the measured peaks}

The score is an extension of a simple peak count: $S_{i}$ of a candidate compound $i$ is calculated based on all fragments $F_{i}$ that explain peaks in the measured spectrum and the bond dissociation energy (BDE) calculated during the in silico fragmentation:

$$
S_{i}=\frac{1}{\max (w)} w_{i}-\frac{1}{2 \max (e)} e_{i}
$$

where

$$
\begin{aligned}
w_{i} & =\sum_{f \in F_{i}}\left(\text { int }_{f}\right)^{0.6} \cdot\left(\text { mass }_{f}\right)^{3} \\
e_{i} & =\frac{1}{\left|F_{i}\right|} \sum_{f \in F_{i}} \sum_{b \in B_{f}} \mathrm{BDE}_{b}
\end{aligned}
$$

In general a peak with a high mass and intensity is more characteristic than peaks with lower mass and intensity. This is reflected by the weighted peak count $w_{i}$, as already proposed by $[3,15]$. The exponents $m=0.6$ and $n=3$ we use are taken from the literature [15]. The weights $w_{i}$ are scaled by $\max (w)$ such that it is between 0 and 1 . We also take the bond dissociation energy (BDE) into account, the higher the BDE, the less likely we consider a fragment. We use the standard enthalpy change upon bond fragmentation from literature, see e.g. [16]. For each candidate $f$ we sum up $\mathrm{BDE}_{b}$ for all bonds $B_{f}$ cleaved along the fragmentation tree for the explained fragments $F_{i}$. Afterwards, for each candidate the arithmetic mean $e_{i}$ of these BDEs is scaled by 2 $\max (e)$ such that it is between 0 and 0.5 .

\section{Neutral loss rules account for rearrangements}

The ionised molecules typically have a single charge. After the fragmentation, the charge remains with either of the resulting fragments, the other is neutral. Because only charged ions can be measured, the mass difference between the two charged ions before and after the fragmentation is referred to as the "neutral loss" [17].

One example of a common neutral loss is $\mathrm{H}_{2} \mathrm{O}$, which is not a true substructure of any molecule. Instead, $\mathrm{H}_{2} \mathrm{O}$ is formed after a hydroxyl group $(\mathrm{OH})$ and a single $\mathrm{H}$ are split off at different (though usually nearby) positions (see Figure 3, where the distance is three). Because individual $\mathrm{H}$ atoms are not considered during the in silico fragmentation, the resulting fragment would never be found without special treatment. MetFrag is checking for structural patterns that can lead to such a non-topological fragmentation. We check within a specified topological distance of the $\mathrm{OH}$-group for another hydrogen and remove both $\mathrm{OH}$ and $\mathrm{H}$.

This non-topological fragmentation is handled by the rules shown in Table 1, other neutral losses are covered by the bond-disconnection approach. Rules can be added easily, e.g. if the compounds measured belong to 


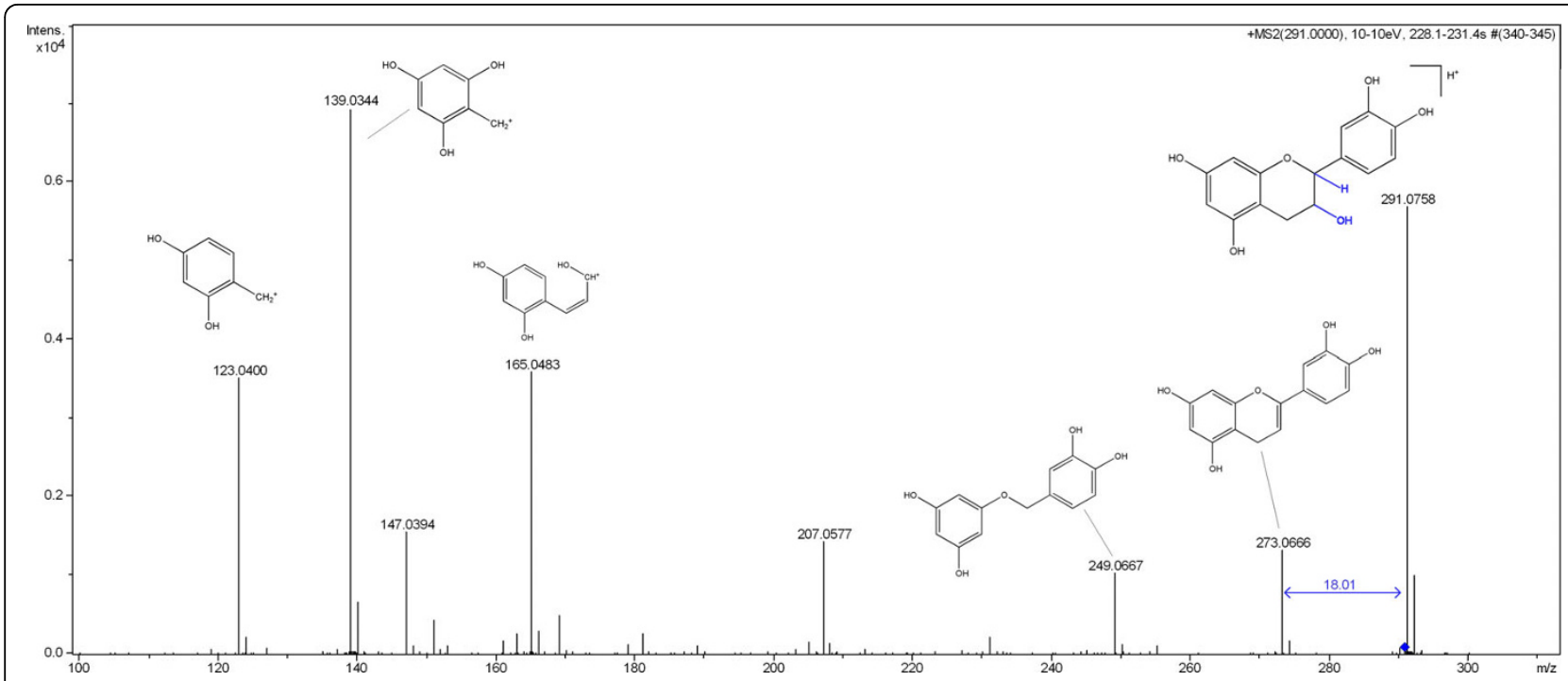

Figure 3 Annotated tandem MS spectrum of Epicatechin. This spectrum for Epicatechin was measured on a Bruker-micrOTOFQ mass spectrometer and manually annotated by an expert. The measured peaks and corresponding fragments for the major signals are depicted. In addition, the non-topological water loss is highlighted in blue.

Table 1 Neutral loss rules

\begin{tabular}{ccccc}
\hline Ion Mode $^{\boldsymbol{a}}$ & Exact Mass $^{\boldsymbol{b}}$ & Topological Fragment $^{\boldsymbol{c}}$ & Neutral Loss $^{\boldsymbol{d}}$ & Maximum Distance $^{\boldsymbol{e}}$ \\
\hline+- & 18.0106 & $\mathrm{OH}$ & $\mathrm{H} 2 \mathrm{O}$ & 3 \\
+- & 27.0109 & $\mathrm{CN}$ & $\mathrm{HCN}$ & 3 \\
+- & $\mathrm{NH} 2$ & $\mathrm{NH} 3$ & 3 \\
+- & $\mathrm{COH}$ & $\mathrm{CH} 2 \mathrm{O}$ & 3 \\
+ & 30.0106 & $\mathrm{COOH}$ & $\mathrm{HCOOH}$ & 3 \\
\hline
\end{tabular}

These rules are applied to the initial candidate structures to account for rearrangements during the tandem MS fragmentation, i.e. neutral losses of unconnected fragments: ${ }^{a}$ ionisation mode where this rule can be applied, ${ }^{b}$ exact mass in Da of the neutral loss, cmolecular formula of the characteristic fragment, ${ }^{d}$ all atoms that are removed, e maximum number of bonds traversed to match neutral loss.

unusual compound classes. MetFrag reads these during start up and applies the rules to the initial candidates, resulting in new (derived) candidate molecules.

\section{Elimination of redundant fragments}

We implemented three alternative structure redundancy checks. Intuitively, a proper graph isomorphism check is the best approach to eliminate structures with the same molecular connectivity. In practice, graph isomorphism checks are not fast enough to process thousands of structures in reasonable time.

Alternatively we implemented an atom based redundancy check: each atom is labelled with a unique identifier and resulting fragments are compared to others based on atom IDs. This method will not detect the redundancy as in $A B A$ mentioned above, because the atoms in the two identical substructures $A$ carry different IDs. This method showed the same identification rate at much lower runtime requirements. To reduce the complexity of the test even further, the molecular formula redundancy check was introduced, which compares fragments based only on their elemental composition. This check will detect the $A B A$ redundancy, but will produce false positives if two structures have the same elemental composition, but with different bond structure, i.e. connectivity. If two fragments have the same molecular formula, the one that requires the lower bond dissociation energy is chosen. This way the fragments which are more likely to occur are considered. The molecular formula redundancy check is used by default, because the results are comparable at considerably reduced runtime.

\section{Structure clustering}

Depending on the upstream database, the MetFrag result list can contain many similar structures or stereo isomers which have identical MetFrag scores. Therefore, we cluster the hits with tied ranks using the pairwise Tanimoto [18] distance of the molecular fingerprints, as implemented in the CDK [11]. All hits with a pairwise similarity $\geq 0.95$ are collapsed into one cluster. 


\section{User interface and available APIs}

Our MetFrag application features an user friendly web interface, http://msbi.ipb-halle.de/MetFrag/. The required input includes the tandem MS peaklist with intensities (Figure 4, top left), selection of the upstream compound database and respective search parameters (top right). Alternatively, a list of database IDs can be provided explicitly. This allows e.g. to select the candidates based on their occurrence in specific pathways. Figure 4 also shows the results browser. A feedback form allows to store all input data, user rating of the hypotheses, and further comments. This helps to collect user-provided test- and training data. Spectra will not be saved unless explicitly granted. The web interface is based on Java Server Faces (JSF) [19], using the Apache MyFaces [20] implementation, ICEfaces [21] (a component library with AJAX capabilities) in an Apache Tomcat [22] servlet container. Thus, MetFrag is platform independent and accessible using most javascript enabled browsers.

We also provide a BioMoby [23] web service, which can be called from other software, including the Taverna workflow engine. Finally, the actual MetFrag algorithms are available as Java library, which can be used to perform batch searches and evaluation.

\section{Results and Discussion}

In this section we give an example of MetFrag results for an exemplary compound, and describe the full test data sets and evaluation criteria. We evaluate MetFrag on two data sets, measured on different instruments, using either KEGG or PubChem as compound library.

For the evaluation we use the merged spectra from different collision energies of compounds where the database id is known. If MetFrag returns multiple hypotheses with tied ranks, we report the most pessimistic position: even if the correct solution has the highest observed score, if 9 other candidates also have the same score, then we assign rank 10.

In addition to the worst case rank we report the cluster rank. Clusters of compounds having a structural Tanimoto similarity $\geq 0.95$ are collapsed and treated as one compound cluster. Again, this measure is quite conservative, because ranks are collapsed only within results having identical scores, and still the worst case cluster rank is reported. The standard deviation of both the raw and cluster ranks for a larger benchmark data set can be quite high, therefore we report not only the average rank, but also the median and $75 \%$ quantile.

\section{Example: Spectrum of Naringenin}

As an example we show the analysis of a tandem MS spectrum of Naringenin $\left(\mathrm{C}_{15} \mathrm{H}_{12} \mathrm{O}_{5}\right.$, KEGG C00509) with MetFrag. Using KEGG as compound library with a realistic $10 \mathrm{ppm}$ window around the exact mass of 272.068 Da will return 15 hits. Each candidate structure is retrieved and fragmented as described in the previous section.

After scoring each structure, the first three results can be seen in Figure 4. The details window shows the fragments that can be explained by the spectrum. The same query in PubChem yields 736 candidates, and Figure 5 shows the 9 top ranked solutions, including the correct compound at worst case rank 8 . The similarity would collapse the isomers into two clusters, resulting in a cluster rank 5 .

\section{Benchmark data sets}

Two data sets were used for evaluation, together consisting of 710 spectra of 151 known compounds. Current instruments allow the acquisition of so called ramp spectra, which combine a range of collision energies in one measurement. In both data sets the compounds were measured at different collision energies. Depending on the compound, informative fragmentation might occur only at higher energies. For other compounds, even low collision energies can lead to a very high degree of fragmentation. For this reason we use composite spectra: two peaks $p_{1}$ and $p_{2}$ from different collision energies are merged $\overline{\mathrm{mz}}=\operatorname{avg}\left(\mathrm{mz}_{1}, \mathrm{mz}_{2}\right)$ if $\left|\mathrm{mz}_{1}-\mathrm{mz}_{2}\right|$ $\leq 0.01 \mathrm{Th}$, retaining the higher intensity $\max \left(i n t_{1}, i n t_{2}\right)$.

\section{Data set I with compound library KEGG}

The first data set consists of 200 spectra from 49 compounds obtained on the API QSTAR Pulsar I in positive mode at several different collision energies, e.g. 10, 20, 30 and $40 \mathrm{eV}$. The spectra were measured at the IPB and are publicly available in the MassBank database http://msbi.ipb-halle.de/MassBank/, see additional file 1 for a list of accession numbers.

MetFrag was used to identify the compounds using the 49 composite spectra within KEGG. Fragments are generated until a tree depth of two is reached. The instrument specific deviation was set to mzabs $=0.01$ and mzppm $=50$.

The initial list of candidates obtained from KEGG contained on average 10.3 compounds. The correct compound has a median of 3 in the MetFrag result list. 25 of the correct compounds were ranked in the top 3 hits and 11 of these are ranked first. MetFrag is a great improvement over a mass-only library search. With 16 021 entries KEGG is a comparably small library. However, the compounds are highly relevant to metabolomics research.

\section{Data set II searched against PubChem}

For the second data set we used the PubChem database, with a much larger collection of natural and synthetic compounds. A collection of 102 compounds with an average mass of $372.5 \mathrm{Da}$ has been measured on a 


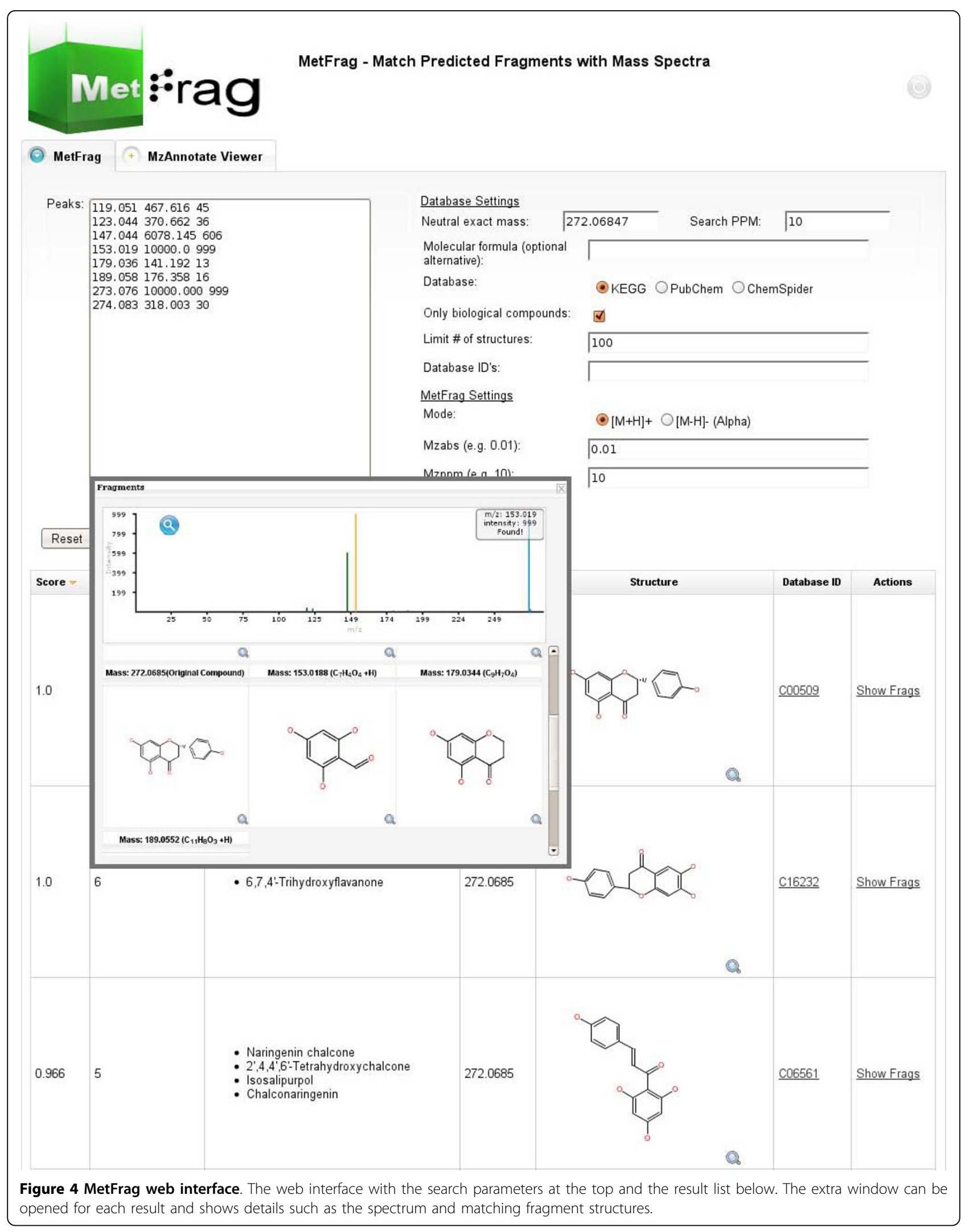




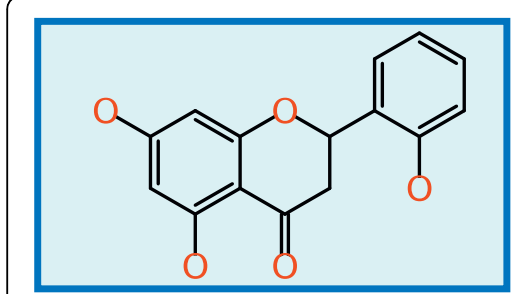

CID: 179999 (Peaks: 6)

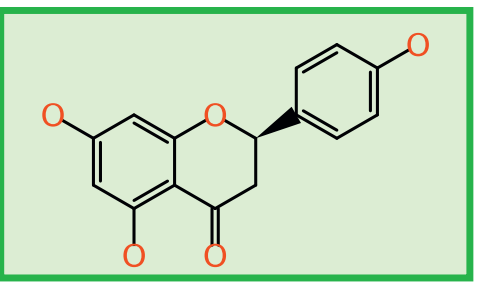

CID: 439246 (Peaks: 6)

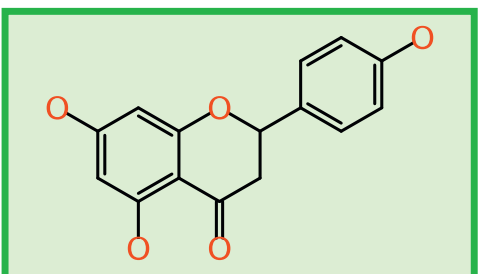

CID: 932 (Peaks: 6)

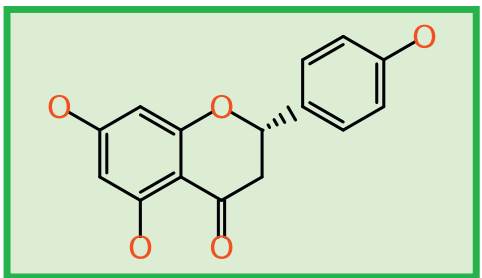

CID: 667495 (Peaks: 6)

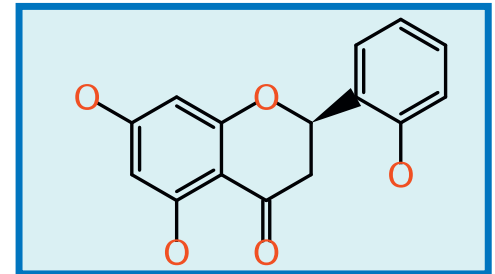

CID: 13889010 (Peaks: 6)

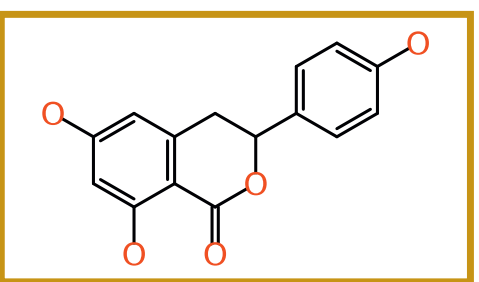

CID: 10333412 (Peaks: 6)

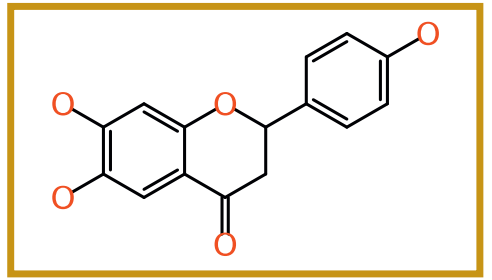

CID: 23724670 (Peaks: 6)

Figure 5 Top candidates for Naringenin against PubChem. The 9 top ranked compounds where the correct solution (CID 932) is reported at (tied) rank 8. Two clusters of structures (green and blue) are identical apart from their stereochemistry, the remaining three structures (yellow) that explain all six tandem MS peaks have a Tanimoto similarity $<0.95$. After clustering with a similarity $\geq 0.95$ the stereoisomers are collapsed into one cluster, resulting in a cluster rank 5 for the correct solution.

Micromass Q-TOF II in positive mode and published by Hill et al. in [8]. Each compound was measured at five different collision energies: 10, 20, 30, 40 and $50 \mathrm{eV}$, for an overall of 510 spectra. All spectra are available from MassBank as well, see additional file 2 for a list of accession numbers. For the spectra from this instrument we used $10 \mathrm{ppm}(\mathrm{mzabs}=0)$ as mass deviation and a maximum tree depth of two. Based on a PubChem snapshot (June 2009) we retrieved on average 2508 candidate compounds.

After the MetFrag scoring, the correct candidate occurred at median rank 31.5 , with the structure clustering the median decreased to 14.5 . The complete results are shown in additional file 2 .
We were also interested in the effect of a larger tree depth: raising the tree depth to three increases the average runtime 5 -fold, and worse, the prediction accuracy decreases. The median of the correct compound degraded to 39 (cluster rank 18). This behaviour can be explained with the positive predictive value (PPV):

$$
P P V=\frac{\mathrm{TP}}{\mathrm{TP}+\mathrm{FP}}
$$

where

$T P=$ peaks explained by correct compound

$F P=$ peaks explained by other candidates. 
The more (smaller) fragments are generated, the more peaks can be matched, which leads to more false positive hits. This dependency is the reason to include the exponent $\operatorname{mass}_{f}^{3}$ in the scoring function. The higher number of false positives results in a PPV of only 0.017 (tree depth three) versus 0.028 using tree depth of two.

Similarly, we applied the neutral loss rules (Table 1) to every generated fragment, not just the initial candidates. Again, we obtained more matching fragments, and the PPV decreased from 0.028 to 0.017 , with an even higher median of the correct compound cluster of 67 .

Another aspect of the evaluation was to use individual spectra instead of the composite spectra. MetFrag showed a poor performance resulting in a median of 43 using $10 \mathrm{ppm}$. An interesting observation is that the median improved to 39.5 if the allowed mass deviation is increased from $10 \mathrm{ppm}$ to $20 \mathrm{ppm}$. Because the merging (and averaging) of peaks in the composite spectra usually results in a more accurate mass, some peaks in individual spectra with a deviation beyond $10 \mathrm{ppm}$ are only matched after relaxing the allowed error window to $20 \mathrm{ppm}$.

Finally, we interpreted some of the cases where MetFrag did not return good results. Table 2 shows many top 10 hits, but also several cases where MetFrag is not able to rank the correct compound even among the top 100. Some of the problematic compounds are Ormetoprim, Strychnine $\mathrm{N}$-oxide and Tetramisole. One reason is the high number of very similar candidate structures, and the difficulty to distinguish them based on the predicted spectra. Another example where many similar structures occur is Tetracycline, but here the rather high rank decreased from 92 to cluster rank 10. Even these large result lists with many similar entries will still give a very good estimation of the possible compound class, which simplifies the subsequent (manual) interpretation and identification.

We also evaluated data set I (measured on the API QSTAR Pulsar I) against PubChem 2009. Because this older mass spectrometer has a much lower mass accuracy than the Micromass Q-TOF II, both the candidate search and the scoring found more false positive matches. Within the 3896 (average) candidates, the median of the correct solution is only 91 . This leads to the conclusion that a good mass accuracy of $\leq 10 \mathrm{ppm}$ is required. Almost all current QTOF instruments are specified at $5 \mathrm{ppm}$ or less, and even higher accuracies are available from Orbitrap or FTICR-MS instruments.

\section{Comparison between MetFrag and MassFrontier}

In their paper [8] Hill et al. evaluate the prediction performance of MassFrontier 4.0 with an approach similar to MetFrag, using PubChem (in the version from February 2006, with $6 \cdot 10^{6}$ entries) as compound database. We added a constraint to our candidate search to include only compounds added in or before February 2006. Our simulated PubChem snapshot returns on average 338 candidates, the previous study only 272 structures. Nevertheless, we use following results to compare MetFrag and MassFrontier. Both MetFrag and the search procedure by Hill consider only compounds containing the elements CHNOPS and ignore molecules which consist of $\mathrm{C}, \mathrm{H}$ only. The previous study reports two separate evaluation strategies: the first combines the automatic ranking with the manual a-posteriori selection of the best spectrum, obtaining the correct result on a median rank 2.5. In practice, this knowledge will not be readily available. The more realistic results are presented in the supplementary material of [8], where a heuristic was used to select one spectrum per compound. The heuristic rule chooses the spectrum with the lowest collision energy which has at most $22 \%$ of the precursor ion intensity. In this case the median drops to $4\left(3^{\text {rd }}\right.$ quantile at 17.5).

The median for MetFrag is 8 ( $3^{r d}$ quantile at 19), and decreases to 4 ( $3^{\text {rd }}$ quantile at 11.75 ) if the $95 \%$ similarity criterion is used. If the results are compared in more detail, this improvement is significant $(p=0.01)$, tested with a one-tailed, paired Wilcoxon signed rank test. The results for both systems are available as additional file 3 .

It would be interesting to evaluate the MassFrontier approach with composite or ramp spectra, where neither automatic nor manual spectra selection would be required.

\section{Empirical runtime evaluation}

The naive and recursive bond-disconnection approach has very high theoretical complexity. We evaluated the real-world runtime by sampling 5900 compounds (unrelated to the test sets) from PubChem with a mass between 100 and $1000 \mathrm{Da}$. In metabolomics research, only few compounds exceed a mass of 1000. Each compound was fragmented (minimum fragment mass 30 Da) to a given tree depth of two and three. Figure 6 shows the runtime of MetFrag on a PC with Intel Q9400 CPU at $2.66 \mathrm{Ghz}$ and $8 \mathrm{~Gb}$ RAM with Ubuntu 8.04, and JVM Sun Java 1.6.0_16-b01. Each point shows the time needed to compute all fragments above $30 \mathrm{Da}$. The yellow and red lines show the non-linear runtime for tree depth two (on average $0.2 \mathrm{~s}$ ) or three (on average 3.4s), respectively. In practice a tree depth of two has the best prediction accuracy (see above) and is fast enough to analyse compounds on demand, even with masses up to $1000 \mathrm{Da}$.

\section{Conclusions}

We have presented an algorithm which is able to identify small molecules from tandem MS measurements among a large set of candidate structures. The scoring function 
Table 2 Results for data set II searched against PubChem

\begin{tabular}{|c|c|c|c|c|c|}
\hline Compound & Candidates & MassFrontier Rank & Candidates & MetFrag Rank & Cluster Rank \\
\hline Thioridazine & 849 & 1 & 1091 & 1 & 1 \\
\hline Bumetanide & 619 & 10 & 768 & 1 & 1 \\
\hline Piperacetazine & 494 & 1 & 626 & 1 & 1 \\
\hline Sufentanil & 445 & 1 & 512 & 1 & 1 \\
\hline Diphenoxylate & 333 & 4 & 369 & 1 & 1 \\
\hline Tetracaine & 308 & 22 & 362 & 1 & 1 \\
\hline Remifentanil & 246 & 1 & 286 & 1 & 1 \\
\hline Hydroxybutorphanol & 180 & 2 & 201 & 1 & 1 \\
\hline Alfentanil & 134 & 1 & 162 & 1 & 1 \\
\hline Etamiphylline & 100 & 3 & 104 & 1 & 1 \\
\hline Ergoloid Mesylate & 7 & 1 & 10 & 1 & 1 \\
\hline Gallamine & 10 & 1 & 8 & 1 & 1 \\
\hline Thonzide & 4 & 1 & 4 & 1 & 1 \\
\hline Spectinomycin & 310 & 1 & 361 & 2 & 1 \\
\hline Methionine Enkephalin & 66 & 1 & 68 & 2 & 1 \\
\hline Leucine Enkephalin & 53 & 2 & 60 & 2 & 1 \\
\hline Dihydroergotamine & 35 & 1 & 38 & 2 & 1 \\
\hline Thiothixene & 726 & 1 & 909 & 3 & 1 \\
\hline Etodolac & 420 & 1 & 580 & 3 & 1 \\
\hline Prednisolone Tebutate & 143 & 4 & 165 & 3 & 1 \\
\hline Oxybutynin & 114 & 6 & 156 & 3 & 1 \\
\hline Apramycin & 54 & 1 & 60 & 3 & 1 \\
\hline Tenoxicam & 28 & 1 & 34 & 3 & 1 \\
\hline Vecuronium & 3 & 1 & 4 & 3 & 1 \\
\hline Methylergonovine & 515 & 1 & 629 & 6 & 1 \\
\hline Rolitetracycline & 105 & 1 & 151 & 6 & 1 \\
\hline Oxytetracycline & 483 & 4 & 614 & 11 & 1 \\
\hline Tetracycline & 529 & 5 & 673 & 19 & 1 \\
\hline Thiethylperazine & 569 & 2 & 671 & 2 & 2 \\
\hline Acetophenazine & 435 & 1 & 546 & 2 & 2 \\
\hline Mebeverine & 96 & 2 & 112 & 2 & 2 \\
\hline Salmeterol & 32 & 1 & 37 & 2 & 2 \\
\hline Terfenadine & 34 & 1 & 35 & 2 & 2 \\
\hline Boldenone Undecylenate & 21 & 2 & 32 & 2 & 2 \\
\hline Buspirone & 36 & 1 & 31 & 2 & 2 \\
\hline Gingerol & 182 & 2 & 195 & 3 & 2 \\
\hline Betaxolol & 190 & 5 & 259 & 4 & 2 \\
\hline Fenoterol & 370 & 5 & 521 & 6 & 2 \\
\hline Taurocholate & 59 & 4 & 65 & 9 & 2 \\
\hline Aminophylline & 94 & 21 & 176 & 3 & 3 \\
\hline Sulfadimethoxine & 94 & 18 & 145 & 3 & 3 \\
\hline Adiphenine & 623 & 6 & 796 & 4 & 3 \\
\hline Perindopril & 102 & 2 & 119 & 6 & 3 \\
\hline Sulfasalazine & 106 & 5 & 116 & 6 & 3 \\
\hline Anileridine & 563 & 251 & 668 & 7 & 3 \\
\hline Prednisolone & 269 & 13 & 363 & 8 & 3 \\
\hline Adenosine Diphosphate & 32 & 3 & 46 & 9 & 3 \\
\hline$\vdots$ & $\vdots$ & $\vdots$ & $\vdots$ & $\vdots$ & \\
\hline Tetramisole & 120 & 1 & 123 & 85 & 79 \\
\hline Oxaprozin & 461 & 101 & 607 & 143 & 94 \\
\hline
\end{tabular}


Table 2: Results for data set II searched against PubChem (Continued)

\begin{tabular}{|c|c|c|c|c|c|}
\hline Antipyrine & 306 & 97 & 341 & 122 & 104 \\
\hline Mefenamic Acid & 579 & 328 & 633 & 146 & 124 \\
\hline Strychnine & 664 & 575 & 882 & 259 & 171 \\
\hline Dimefline & 644 & 644 & 876 & 294 & 175 \\
\hline Ormetoprim & 270 & 124 & 317 & 233 & 191 \\
\hline Strychnine N-oxide & 1185 & 1098 & 1672 & 1012 & 618 \\
\hline Average: & $272.2( \pm 24.2)$ & $44.2( \pm 14.1)$ & $338.4( \pm 31.5)$ & $34.2( \pm 10.9)$ & $21.6( \pm 6.8)$ \\
\hline Median: & 183.5 & 4 & 231.5 & 8 & 4 \\
\hline 75\% Quantile: & 431.3 & 17.5 & 518.8 & 19 & 11.8 \\
\hline Std. Deviation: & 244.1 & 142.4 & 318.1 & 109.8 & 69.1 \\
\hline
\end{tabular}

The results on the left were reported in [8]. The corresponding MetFrag results are on the right where the candidate search was restricted to the PubChem as of February 2006 (we retrieved slightly more candidates than reported by Hill et. al.). Only the best 47 and eight worst Metfrag results are shown, the full table is given as additional file 3 .

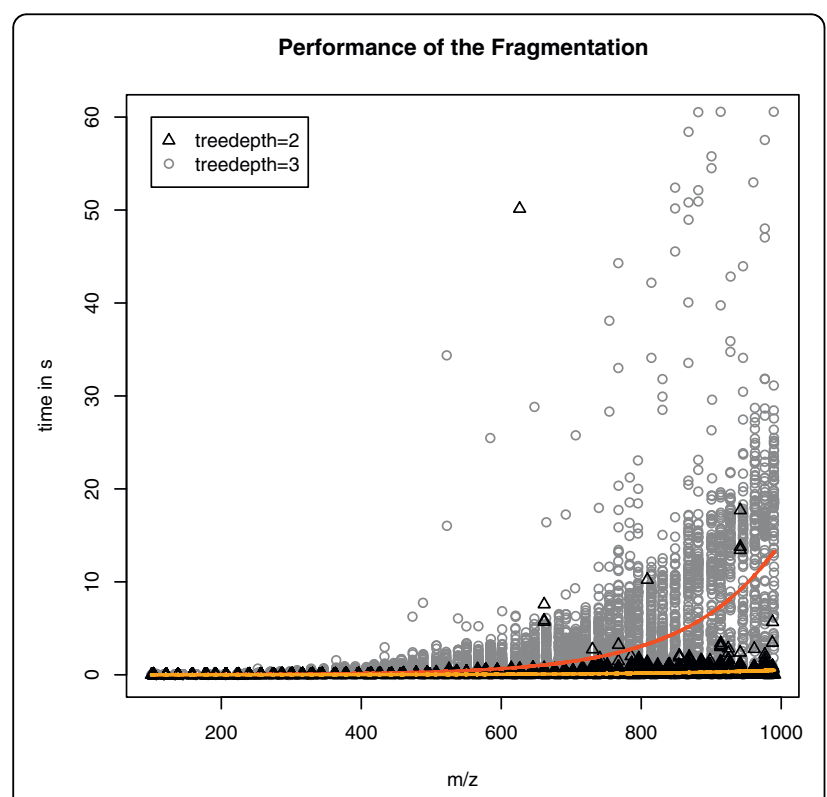

Figure 6 Empirical runtime. Runtime for the in silico fragmentation step on 5900 compounds randomly drawn from PubChem, with uniform mass distribution between 100 and 1000 Da. Limiting the tree depth of the in silico fragmentation to two (orange) results in an average runtime of $0.2 \mathrm{~s}$ for one compound. The exponential runtime can be seen especially when a larger tree depth (red) is used, raising the runtime to $3.4 \mathrm{~s}$.

does not require a set of fragmentation reactions or an actual simulation of the fragmentation process. MetFrag is able to query KEGG, PubChem and ChemSpider, and local databases can be integrated with little effort.

In comparison to the system described in [8] (which included human expertise), MetFrag achieves better results than MassFrontier.

For dedicated metabolite databases such as KEGG, the correct identification is generally among the first few candidates. Given the sheer size of generic compound libraries such as PubChem, it is no surprise that the result lists contain many structurally highly similar compounds. Hence, an unambiguous identification is generally not possible, but usually the compound class can be derived from the results. A principal limitation is the inability to distinguish stereoisomers which is not possible from MS data alone. The final identification according to MSI recommendations [24] requires the comparison against spectra of authentic standards, or even complementary analysis methods such as NMR.

Our tool MetFrag improves the identification of unknown substances from tandem MS spectra. It is fast enough to be used in the interactive web application, and has a user-friendly interface and result browser.

\section{Availability and Requirements}

- Project home page: http://metware.org/

- Operating system(s): Platform independent

- Programming language: Java

- Other requirements: Java $\geq 1.6$, Tomcat $\geq 6.0$

- License: GNU LGPL v3 (or later)

Additional file 1: MassBank_KEGG_results. Full list of mass spectra and compounds used in section "Data set I searched against KEGG". This includes accession numbers in the MassBank system. For each compound the number of candidates and the rank of the correct solution is given.

Additional file 2: HillData_PubChem2009. Full list of mass spectra and compounds used in section "Data set II searched against PubChem". This includes accession numbers in the MassBank system. For each compound the number of candidates and the rank of the correct solution is given.

\section{Additional file}

3: Comparison_MassFrontier_MetFrag_PubChem2006. This file includes the full results from table 2 in section "Data set II searched against PubChem". The candidate search was restricted to the PubChem as of February 2006. For convenience, we also include the results reported in [8].

\section{Acknowledgements}

We thank the CDK team (especially Egon Willighagen and Rajarshi Guha) for this cheminformatics library, and Michael Gerlich, Carsten Kuhl and Ralf 
Tautenhahn for helpful discussions. SS is funded by the BMBF (GABI-ProTect 0315051C).

\section{Author details}

'Leibniz Institute of Plant Biochemistry- Department of Stress- and Developmental Biology, Weinberg 3, 06120 Halle(Saale), Germany. ${ }^{2}$ Institut für Informatik, Martin-Luther-Universität, Halle-Wittenberg, Von-Seckendorffplatz 1, 06120 Halle (Saale), Germany

\section{Authors' contributions}

SW implemented the MetFrag application, web interface and performed the evaluation. SS provided the MS expertise, MM-H and SN provided input for the requirements, the algorithmic design and architecture. All authors contributed to, read and approved the final manuscript.

Received: 24 November 2009 Accepted: 22 March 2010

Published: 22 March 2010

\section{References}

1. Dunn WB: Current trends and future requirements for the mass spectrometric investigation of microbial, mammalian and plant metabolomes. Physical Biology 2008, 5:011001, (24pp).

2. Kopka J, Schauer N, Krueger S, Birkemeyer C, Usadel B, Bergmuller $E_{i}$ Dormann P, Weckwerth W, Gibon Y, Stitt M, Willmitzer L, Fernie AR, Steinhauser D: GMD@CSB.DB: the Golm Metabolome Database. Bioinformatics 2005, 21(8):1635-1638.

3. Horai H, Arita M, Nishioka T: Comparison of ESI-MS Spectra in MassBank Database. BioMedical Engineering and Informatics, 2008. BMEl 2008. International Conference on 2008, 2:853-857.

4. Smith CA, Maille GO, Want EJ, Qin C, Trauger SA, Brandon TR, Custodio DE, Abagyan R, Siuzdak G: METLIN: A Metabolite Mass Spectral Database. Proceedings of the 9th International Congress of Therapeutic Drug Monitoring and Clinical Toxicology Louisville, Kentucky 2005, 27:747-751.

5. ACD/MS Fragmenter. [http://www.acdlabs.com/products/adh/ms/ms_frag/]

6. Mass Frontier. [http://www.highchem.com/]

7. Heinonen M, Rantanen A, Mielikäinen T, Kokkonen J, Kiuru J, Ketola RA, Rousu J: FiD: a software for ab initio structural identification of product ions from tandem mass spectrometric data. Rapid Communications in Mass Spectrometry 2008, 22(19):3043-3052.

8. Hill DW, Kertesz TM, Fontaine D, Friedman R, Grant DF: Mass Spectral Metabonomics beyond Elemental Formula: Chemical Database Querying by Matching Experimental with Computational Fragmentation Spectra. Analytical Chemistry 2008, 80(14):5574-5582.

9. Heinonen M, Rantanen A, Mielikäinen T, Pitkanen E, Kokkonen J, Rousu J: $\mathrm{Ab}$ initio prediction of molecular fragments from tandem mass spectrometry data. German Conference on Bioinformatics 2006, 40-53.

10. Hill AW, Mortishire-Smith RJ: Automated assignment of high-resolution collisionally activated dissociation mass spectra using a systematic bond disconnection approach. Rapid Communications in Mass Spectrometry 2005, 19(21):3111-3118.

11. Steinbeck C, Han Y, Kuhn S, Horlacher O, Luttmann E, Willighagen E: The Chemistry Development Kit (CDK): An Open-Source Java Library for Chemo- and Bioinformatics. Journal of Chemical Information and Computer Sciences 2003, 43(2):493-500.

12. KEGG Compound. [http://www.genome.jp/kegg/compound/]

13. PubChem. [http://pubchem.ncbi.nlm.nih.gov/].

14. Chemspider. [http://www.chemspider.com/].

15. Stein SE, Scott DR: Optimization and testing of mass spectral library search algorithms for compound identification. Journal of the American Society for Mass Spectrometry 1994, 5(9):859-866.

16. Luo Y: Handbook of bond dissociation energies in organic compounds Boca Raton, CRC Press 2003

17. Gross JH: Mass Spectrometry: A Textbook Springer, Berlin, 1 2004, corr. 2nd printing edition 2004.

18. Butina D: Unsupervised Data Base Clustering Based on Daylight's Fingerprint and Tanimoto Similarity: A Fast and Automated Way To Cluster Small and Large Data Sets. Journal of Chemical Information and Computer Sciences 1999, 39(4):747-750.

19. Java Server Faces. [http://java.sun.com/javaee/javaserverfaces/]

20. Apache My Faces. [http://myfaces.apache.org/core12/index.html].

21. ICEfaces. [http://www.icefaces.org/].
22. Apache Tomcat 6. [http://tomcat.apache.org/]

23. Biomoby. [http://www.biomoby.org/].

24. Sumner LW, Amberg A, Barrett D, Beale M, Beger R, Daykin C, Fan T, Fiehn O, Goodacre R, Griffin JL, Hankemeier T, Hardy N, Harnly J, Higashi R, Kopka J, Lane A, Lindon JC, Marriott P, Nicholls A, Reily M, Thaden J, Viant MR: Proposed minimum reporting standards for chemical analysis. Metabolomics 2007, 3(3):211-221.

doi:10.1186/1471-2105-11-148

Cite this article as: Wolf et al: In silico fragmentation for computer assisted identification of metabolite mass spectra. BMC Bioinformatics 2010 11:148.

\section{Submit your next manuscript to BioMed Central and take full advantage of:}

- Convenient online submission

- Thorough peer review

- No space constraints or color figure charges

- Immediate publication on acceptance

- Inclusion in PubMed, CAS, Scopus and Google Scholar

- Research which is freely available for redistribution

Submit your manuscript at www.biomedcentral.com/submit
C Biomed Central 